\title{
ASOCIACIÓN ENTRE EL RIESGO DE DEPRESIÓN MAYOR Y EL BAJO NIVEL DE ACTIVIDAD FÍSICA EN TRABAJADORES PERUANOS QUE CURSAN ESTUDIOS UNIVERSITARIOS
}

\author{
Luis Murillo-Pérez ${ }^{1, a}$, Carolay Rojas-Adrianzén ${ }^{1, a}$, Gabriela Ramos-Torres ${ }^{1, a}$, Bryan Cárdenas-Vicente ${ }^{1, a}$, \\ Wendy Hernández-Fernández ${ }^{1, a}$, Piero Larco-Castilla1,a, Luis Haro-García ${ }^{2,3, b}$, \\ Edward Mezones-Holguín ${ }^{1,4, c}$
}

\begin{abstract}
RESUMEN
Con el objetivo de evaluar si existe asociación entre el riesgo de depresión mayor (RDM) y la actividad física (AF) controlado por variables sociodemográficas y académicas en trabajadores que cursan estudios de pregrado en una universidad privada de Lima, se desarrolló un estudio de corte transversal analítico en 1111 personas. Se utilizó el inventario de depresión mayor y el cuestionario internacional de actividad física, para medir RDM y AF, respectivamente. La prevalencia de RDM fue 4,2\%. En el modelo de regresión de múltiples variables - ajustado por edad, sexo, desempleo y horas de sueño- la AF baja se asocia con un incremento de la odds de RDM (OR 2,15; IC 95\%:1,16-4,00). Se concluye que existe asociación entre la RDM y la AF en la población estudiada, la cual es independiente de factores sociodemográficos y académicos. Se sugieren mejorar estrategias de tamizaje y el desarrollo de estudios longitudinales para evaluar causalidad.
\end{abstract}

Palabras clave: Depresión; Actividad motora; Universidades; Trabajadores; Perú (fuente: DECS/BIREME).

\section{ASSOCIATION BETWEEN THE RISK OF MAJOR DEPRESSION AND LOW PHYSICAL ACTIVITY IN PERUVIAN WORKERS STUDYING IN UNIVERSITIES}

\begin{abstract}
In order to assess if an association exists between the risk of major depression (RMD) and physical activity (PA), controlling for demographic and academic variables in workers enrolled in undergraduate studies at a private university in Lima, Peru, we carried out a cross-sectional study of 1,111 people. We used the MajorDepression Inventory (MDI) and the International Physical Activity Questionnaire (IPAQ) to measure RMD and PA, respectively. RMD prevalence was $4.2 \%$. In the multiple regression model adjusted for age, gender, unemployment and hours of sleep, low levels of PA were associated with increased odds of RDM (OR: $2.15,95 \% \mathrm{Cl}: 1.16$ to 4.00 ). We conclude that there is an association between RMD and PA in the study population, independent of demographic and academics factors. Strategies to improve screening and development of longitudinal studies to assess causality are suggested.
\end{abstract}

Key words: Depression; Motor activity; Universities; Workers; Peru (source: MeSH/NLM).

\section{INTRODUCCIÓN}

El trastorno depresivo mayor (TDM) tiene una importante carga de enfermedad a nivel mundial. Se asocia con altos costos y requiere una aproximación multifactorial para su detección, tratamiento y prevención (1). Existen deficiencias en su oportuno diagnóstico y registro, lo cual lo han convertido en un reto para la salud pública (2). Por tanto, su abordaje debe ser de interés prioritario en los sistemas de salud.
Los factores asociados a TDM son múltiples y varían según la población estudiada (1). Se ha descrito una mayor frecuencia en trabajadores ${ }^{(3)}$-lo cual podría acentuarse con el desempleo en momentos de crisis económica- ${ }^{(4)} y$ en estudiantes universitarios que en la población general (5). Además, la exposición simultánea a carga académica y carga laboral -y en algunos casos carga familiar-, y la interacción entre estas, conllevan a una reducción del tiempo para actividades de recreación, ocio y deporte. Consecuentemente, los trabajadores que realizan

Escuela de Medicina, Universidad Peruana de Ciencias Aplicadas (UPC). Lima, Perú.

Departamento de Salud Pública, Facultad de Medicina, Universidad Nacional Autónoma de México (UNAM). Ciudad de México, México.

Unidad de Investigación en Salud del Trabajo, Instituto Mexicano de Seguridad Social (IMSS). Ciudad de México, México.

4 Unidad de Análisis y Generación de Evidencias en Salud Pública (UNAGESP), Centro Nacional de salud Pública, Instituto Nacional de Salud. Lima, Perú.

Estudiante de Medicina; ${ }^{\mathrm{b}}$ Médico cirujano, especialista en Medicina Familiar y Salud del Trabajo, doctor en Ciencias. ${ }^{\mathrm{C}}$ Médico cirujano, maestro en Ciencias en Epidemiología Clínica.

Recibido: : 24-02-14 Aprobado: 11-06-14

Citar como: Murillo-Pérez L, Rojas-Adrianzén C, Ramos-Torres G, Cárdenas-Vicente B, Hernández-Fernández W, Larco-Castilla P, et al. Asociación entre el riesgo de depresión mayor y el bajo nivel de actividad física en trabajadores peruanos que cursan estudios universitarios. Rev Peru Med Exp Salud Publica. $2014 ; 31(3): 520-24$. 
estudios universitarios tendrían mayor probabilidad de TDM y de niveles bajos de actividad física (AF).

La AF se ha asociado a TDM y otras afecciones de la salud mental. Ante mayores niveles de AF, existe una menor probabilidad de desarrollar sintomatología depresiva (SD) ${ }^{(6)}$ en adolescentes y una disminución de SD en adultos jóvenes ${ }^{(7)}$. Una mayor AF se asocia a menor frecuencia de trastorno depresivo en mujeres en edad mediana ${ }^{(8)}$ y en ancianos ${ }^{(9)}$. No obstante, aún existe controversia debido a la identificación de deficiencias en el diseño, análisis y los distintos mecanismos de mediación y causalidad expuestos en las investigaciones ${ }^{(7,10)}$

A pesar de lo expresado, no se han publicado estudios que evalúen dicha asociación en trabajadores peruanos que cursa estudios universitarios, más aun cuando el mercado educativo ofrece programas académicos específicos -con edad promedio superior a quienes cursan el pregrado regular- en horarios compatibles con sus actividades laborales. Esta investigación tuvo como objetivo evaluar si existe asociación entre el riesgo de depresión mayor (RDM) y la AF en una población de trabajadores que cursan estudios universitarios en una universidad privada de Lima, controlado por variables sociodemográficas y académicas.

\section{EL ESTUDIO}

\section{DISEÑO, POBLACIÓN, MUESTRA Y MUESTREO}

Durante el año 2012 se realizó un estudio de corte transversal analítico en la División de Estudios Profesionales para Ejecutivos (EPE) de una universidad privada en Lima-Perú, la cual ofrece formación universitaria de pregrado a personas que trabajan. Se incluyeron estudiantes matriculados en semestre académico I-2012 y se excluyeron a aquellos con alguna discapacidad física, que usaban medicamentos antidepresivos o que se encontraban en periodo de duelo.

Usando el programa nQuery Advisor 6.0® (Statistical Solutions, Cork, Irlanda). Se estimó una muestra mínima de 917 participantes, basados en un estimado para un modelo de regresión logística binaria con un OR 0,64; una fracción de exposición de 33\% y una razón deprimidos/no deprimidos de 0,5 ; con una significancia y potencia estadísticas de 5 y $80 \%$, respectivamente. Con una proporción de rechazo de $15 \%$ en base al estudio piloto, se requirió invitar mínimamente a 1079 participantes. La cantidad de estudiantes por carrera y sede se calcularon sobra la base de una distribución proporcional de acuerdo al reporte de la institución. La selección de cada participante fue no probabilística.

\section{VARIABLES}

Se midieron las variables edad, el sexo, el estado civil, la descendencia, la situación laboral actual (trabaja/no trabaja), el semestre académico, la facultad (Administración/ Negocios/Ingeniería) y la sede universitaria (Monterico/ San Miguel/San Borja). Se evaluaron las horas de descanso por día (horas de sueño), aspectos relacionados al transporte hacia la universidad y el hogar, la frecuencia de consumo de tabaco y el consumo de alcohol (frecuencia y escala de CAGE validada al español).

Se usó la versión corta del cuestionario internacional de actividad física (IPAQ) ${ }^{(11)}$ y la prueba de depresión mayor, ambas validadas al español, para definir baja AF y RDM (con el algoritmo desarrollado por Nebreda \& Aliaga), respectivamente ${ }^{(12)}$.

\section{PROCEDIMIENTOS}

Se realizó una prueba piloto sobre 150 personas. Los cuestionarios impresos y las encuestas fueron revisados tras la aplicación para verificar la respuesta total a los ítems. Se obtuvo consentimiento informado de los participantes. El protocolo fue aprobado por la Cátedra de Metodología de la Investigación de la Escuela de Medicina de la UPC.

\section{ANÁLISIS ESTADÍSTICO}

La base de datos fue generada mediante digitación doble e independiente. Se evaluó la concordancia entre digitadores, y entre 50 fichas impresas y registros digitales. Para el análisis se utilizó STATA® v11.0 (STATA Corporation, College Station, Texas, USA).

En las variables categóricas se usó distribución de frecuencias y porcentajes; y en las numéricas media y desviación estándar, o mediana y desviación intercuartílica (de acuerdo a la normalidad y los valores extremos). La asociación entre las variables numéricas y el RDM se evaluó con la prueba de suma de rangos de Wilcoxon, previa evaluación de la normalidad (Shapiro Wilk) y la homogeneidad de varianzas (Levene). Para testear la asociación entre el RDM y las variables categóricas se utilizó la prueba de chi-cuadrado o el test exacto de Fisher, según los valores esperados.

Se desarrolló un modelo de asociación crudo por medio de una regresión logística binaria, con el RDM como variable de respuesta y la AF como variable de exposición. También se hizo un modelo múltiple reducido incorporando las variables de control significativas $(p<0,05)$ en el bivariado y un modelo de regresión múltiple, en el cual se incluyó las posibles variables de interacción. 
Se ejecutó un análisis de máxima verosimilitud entre ambos modelos, al no encontrarse diferencias $(p>0,2)$ se mantuvo el modelo reducido. La bondad de ajuste se midió con la prueba de Hosmer Lemeshow. Se reportó OR con IC 95\%. Dado que no se cumplió el supuesto de linealidad entre las HDD y el logaritmo del OR, se generó un punto de corte con curva ROC en seis horas de sueño.

\section{HALLAZGOS}

Se incluyeron 1111 participantes (Figura 1), la mayoría eran varones y estudiaban en la Facultad de Administración. El 2,3\% estaba desempleado; el 1,6\% tenía dependencia alcohólica y cerca de la cuarta parte consumía tabaco. El $4,2 \%$ presentaron RDM y el $34,2 \%$ tienen baja AF (Tabla 1 ).

Se observó una mayor proporción de RDM en aquellos que tuvieron baja $\mathrm{AF}(6,3 \%)$ que en aquellos con $\mathrm{AF}$ alta o moderada $(3,2 \%)(p<0,018)$. Se encontró proporciones mayores de RDM en los desempleados (16\%) y en las mujeres $(6,1 \%)$. Asimismo, la edad $(p<0,05)$ y las horas de sueño $(p<0,01)$ fueron menores en los que tenían RDM. No se encontró asociación con otras variables (Tabla 2).

En el modelo crudo, la odds de tener AF baja en quienes tienen RDM es 2,07 veces la odds de tener AF baja en quienes no tienen RDM, lo cual se mantiene ( $O R=2,15$; IC95\%: 1,14-4,0) en el modelo ajustado.

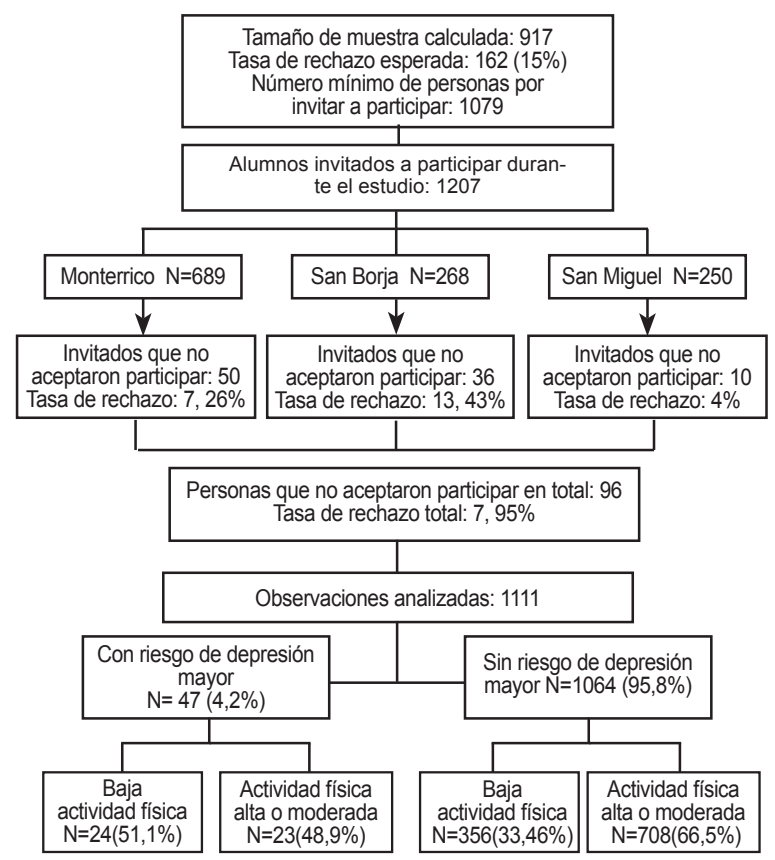

Figura 1. Flujo general de la investigación: cálculo del tamaño muestral, proceso de selección de participantes y resultado general
Tabla1. Características generales de la población en estudio $(n=1111)$

\begin{tabular}{|c|c|}
\hline & $\mathbf{N}(\%)$ \\
\hline Edad (años)* & $29 \pm 4$ \\
\hline Varones & $669(60,2)$ \\
\hline \multicolumn{2}{|l|}{ Estado civil } \\
\hline Soltero & $732(66,0)$ \\
\hline Casado & $246(22,1)$ \\
\hline Viudo & $41(3,7)$ \\
\hline Divorciado & $24(2,2)$ \\
\hline Conviviente & $67(6,0)$ \\
\hline Sin hijos & $750(67,5)$ \\
\hline No trabaja actualmente & $25(2,3)$ \\
\hline \multicolumn{2}{|l|}{ Sede académica } \\
\hline Monterrico & $639(57,5)$ \\
\hline San Borja & $232(20,8)$ \\
\hline San Miguel & $240(21,6)$ \\
\hline \multicolumn{2}{|l|}{ Facultad } \\
\hline Administración & $416(37,4)$ \\
\hline Negocios & $226(20,3)$ \\
\hline Ingeniería & $469(42,2)$ \\
\hline \multicolumn{2}{|c|}{ Lugar desde donde se traslada hacia la universidad } \\
\hline Casa & $114(10,3)$ \\
\hline Trabajo & $826(74,4)$ \\
\hline Otro & $170(15,3)$ \\
\hline Horas diarias de descanso* & $6 \pm 0,5^{*}$ \\
\hline $\begin{array}{l}\text { Tiempo para llegar a la universidad } \\
\text { (minutos) }{ }^{* *}\end{array}$ & $45 \pm 15^{*}$ \\
\hline Tiempo para llegar a la casa (minutos)** & $35 \pm 20^{*}$ \\
\hline \multicolumn{2}{|l|}{ Consumo de alcohol (EBBA) } \\
\hline No consume & $546(49,1)$ \\
\hline Bebedor social & $435(39,1)$ \\
\hline Consumo de riesgo & $60(5,4)$ \\
\hline Consumo perjudicial & $52(4,7)$ \\
\hline Dependencia alcohólica & $18(1,6)$ \\
\hline \multicolumn{2}{|l|}{ Consumo de tabaco } \\
\hline Nunca ha fumado & $501(45,1)$ \\
\hline Dejo de fumar hace $>1$ año & $238(21,4)$ \\
\hline Dejo de fumar hace $<1$ año & $98(8,9)$ \\
\hline Fuma actualmente & $274(24,6)$ \\
\hline Riesgo de depresión mayor & $47(4,2)$ \\
\hline Baja actividad física & $380(34,2)$ \\
\hline
\end{tabular}

En este estudio la AF baja se asocia con un incremento de la odds de RDM, independiente de las variables sociodemográficas y académicas en trabajadores que cursan estudios de pregrado. Esto constituye una de las primeras descripciones de esta asociación en población latina que labora y estudia simultáneamente, y se condicen con evidencia internacional publicada. En trabajadores japoneses se ha descrito una menor frecuencia de SD en quienes tienen AF regular, así como mejoras en la SD en participantes de un programa de AF (13). Asimismo, estudios en adolescentes, adultos, mujeres en edad mediana y adultos mayores tienen resultados similares ${ }^{(6-9)}$ y se ha observado un efecto terapéutico de la AF en pacientes deprimidos ${ }^{(14)}$, el cual podría ser dosis dependiente ${ }^{(15)}$. Aunque, otros autores 
Tabla 2. Factores asociados a riesgo de depresión mayor en la población estudiada (análisis bivariado)

\begin{tabular}{|c|c|c|c|}
\hline & $\begin{array}{c}\text { Con riesgo } \\
\text { de depresión } \\
\text { mayor }=47\end{array}$ & $\begin{array}{c}\text { Sin riesgo de } \\
\text { depresión mayor } \\
=1064\end{array}$ & $\begin{array}{c}\text { Valor } \\
p\end{array}$ \\
\hline & $N(\%)$ & $\mathrm{N}(\%)$ & \\
\hline \multicolumn{4}{|l|}{ Actividad física } \\
\hline $\begin{array}{l}\text { Actividad física } \\
\text { moderada o alta }\end{array}$ & $23(3,2)$ & $708(96,9)$ & $0,018^{*}$ \\
\hline Baja actividad física & $24(6,3)$ & $356(93,7)$ & \\
\hline Edad & $28 \pm 4$ & $30 \pm 4$ & $0,003^{\star *}$ \\
\hline \multicolumn{4}{|l|}{ Sexo } \\
\hline Hombre & $20(3,0)$ & $649(97,0)$ & $0,011^{*}$ \\
\hline Mujer & $27(6,1)$ & $415(94,0)$ & \\
\hline \multicolumn{4}{|l|}{ Número de hijos } \\
\hline Sin hijos & $37(5,0)$ & $713(96,0)$ & $0,093^{*}$ \\
\hline Con hijos & $10(2,8)$ & $351(97,2)$ & \\
\hline \multicolumn{4}{|l|}{ Desempleo } \\
\hline No Trabaja actualmente & $4(16,0)$ & $21(84,0)$ & $0,019^{* * *}$ \\
\hline Trabaja actualmente & $43(3,9)$ & $1043(96,1)$ & \\
\hline \multicolumn{4}{|l|}{ Facultad } \\
\hline Administración & $17(4,1)$ & $399(96,0)$ & $0,106^{*}$ \\
\hline Negocios & $15(6,6)$ & $211(93,4)$ & \\
\hline Ingeniería & $15(3,2)$ & $454(97,0)$ & \\
\hline \multicolumn{4}{|l|}{ Sede } \\
\hline Monterrico & $24(3,8)$ & $615(96,2)$ & $0,619^{*}$ \\
\hline San Borja & $11(4,7)$ & $221(95,3)$ & \\
\hline San Miguel & $12(5,0)$ & $228(95,0)$ & \\
\hline \multicolumn{4}{|l|}{ Traslado a universidad } \\
\hline Desde casa & $5(4,4)$ & $109(95,6)$ & $0,94^{*}$ \\
\hline Desde trabajo & $34(4,1)$ & $792(95,9)$ & \\
\hline Otros & $8(4,7)$ & $162(95,3)$ & \\
\hline $\begin{array}{l}\text { Tiempo para llegar a la } \\
\text { universidad }\end{array}$ & $59(30)$ & $45(30)$ & $0,270^{* *}$ \\
\hline $\begin{array}{l}\text { Tiempo para llegar a } \\
\text { la casa }\end{array}$ & $30(20,0)$ & $35(20,0)$ & $0,570^{* *}$ \\
\hline Horas de sueño & $5 \pm 1,0$ & $6 \pm 0,5$ & $0,001^{* *}$ \\
\hline \multicolumn{4}{|l|}{ Consumo de alcohol } \\
\hline No consume & $22(4,0)$ & $524(96,0)$ & $0,320^{* * *}$ \\
\hline Bebedor social & $18(4,1)$ & $417(95,1)$ & \\
\hline Consumo de riesgo & $1(1,7)$ & $59(98,3)$ & \\
\hline Consumo perjudicial & $4(7,7)$ & $48(92,3)$ & \\
\hline $\begin{array}{l}\text { Dependencia } \\
\text { Alcohólica }\end{array}$ & $2(11,1)$ & $16(88,9)$ & \\
\hline \multicolumn{4}{|l|}{ Consumo de tabaco } \\
\hline Nunca ha fumado & $23(5,0)$ & $478(95,4)$ & $0,350^{*}$ \\
\hline $\begin{array}{l}\text { Dejo de fumar hace } \\
>1 \text { año }\end{array}$ & $6(2,5)$ & $232(97,5)$ & \\
\hline $\begin{array}{l}\text { Dejo de fumar hace } \\
<1 \text { año }\end{array}$ & $3(3,1)$ & $95(97,0)$ & \\
\hline Fuma actualmente & $15(5,5)$ & $259(94,5)$ & \\
\hline
\end{tabular}

${ }^{*} \mathrm{Chi}^{2},{ }^{* *}$ mediana \pm desviación cuartílica. Suma de rangos de Wilcoxon, ${ }^{* \star *}$ Test exacto de Fisher

refieren que aún existe controversia sobre el efecto de la $A F$ en la SD ${ }^{(10)}$. Por tanto, a pesar de que la evidencia epidemiológica favorezca la posibilidad de una relación causal, la plausibilidad subyacente es importante.

Se han propuesto varios mecanismos para explicar la asociación entre la AF y el TDM. Craft\&Perna los agrupan en cinco hipótesis, las cuales podrían clasificarse en biológicas: la termogénesis, la basada en endorfinas y la de las monoaminas; y psicológicas: la de la distracción y la del aumento de la autoeficacia. La termogénesis explica que la generación de calor y el subsecuente incremento de temperatura en algunas regiones del cerebro conllevarían a disminuir la SD al producir relajación muscular. La segunda hipótesis propone que el ejercicio incrementa la liberación de $\beta$-endorfinas, las cuales contribuirían al lograr un mejor estado de ánimo. La hipótesis de las monoaminas - que según estos autores es una de las más desarrolladas expresa que el ejercicio incrementaría la disponibilidad de neurotransmisores cerebrales como la serotonina, la dopamina y la norepinefrina, cuya disminución -sobre todo de la serotonina- está fuertemente relacionada al desarrollo de SD. La hipótesis de la distracción sugiere que el ejercicio funcionaría como un pasatiempo, que contribuiría a disminuir el pensar en los problemas, y en la depresión en sí misma. La hipótesis de la autoeficacia plantea que la persona posee las habilidades necesarias para desarrollar una tarea específica y, cuando estas son logradas, se contribuye a un mayor nivel de confianza y a una disminución de la SD; en ese sentido, al desarrollar la $A F$, se refuerza el hecho de que la persona es autoeficaz y con ello la SD disminuiría. Asimismo, ambos mecanismos psicológicos podrían interactuar en la disminución de la SD (16). También se ha propuesto que el ejercicio incrementa los niveles del factor neutrópico derivado del cerebro a nivel periférico con activación de los receptores canabinoides, explicando así la disminución de la SD ${ }^{(17)}$. Se ha señalado que la adiposidad corporal tendría efecto deletéreo sobre el bienestar psicológico, por tanto, podría mediar el efecto del ejercicio físico, al producir disminución de la grasa corporal ${ }^{(18)}$. Todo ello refuerza lo encontrado en esta investigación.

Este estudio tiene algunas limitaciones. Primero, al ser un análisis transversal no puede establecerse de modo directo una relación causal. No obstante, la magnitud de la asociación producto de un modelo de regresión múltiple, la concordancia de los resultados con lo encontrado en la literatura previa, los antecedentes producto de estudios de intervención, la dosis dependencia reportada y la plausibilidad biológica expresada, contribuirían a pensar en un potencial efecto causal (19). Segunda, no se profundizó en las características de los puestos de trabajo. Pues la AF durante las horas de trabajo disminuye la probabilidad de SD en trabajadores y por otro, los problemas del sueño -asociados a los turnos laborales y un ambiente laboral problemático- se asocian a TDM ${ }^{(20)}$. Sin embargo, se midió la situación laboral actual, donde el desempleo se asocia a mayores odds de RDM y medimos las horas de sueño, donde el dormir menos de seis horas se asociaba a RDM. Estas dos variables fueron consideradas como marcadores del ambiente laboral y, por tanto, fueron parte del ajuste del modelo final. Tercero, la 
medida de las dos variables principales fue realizada mediante cuestionarios autoadministrados y no con una medición directa. Sin embargo, la mayoría de estudios epidemiológicos utilizan cuestionarios para medir TDM o SD (12); asimismo, el IPAQ es un instrumento que ha sido validado en población latina y utilizado en series en nuestro continente ${ }^{(11)}$. Cuarto, la selección final de los participantes fue no probabilística y no se cuantificó la medicación antidepresiva en los que rechazaron participar, lo cual podría generar un sesgo de selección. Aunque, al tratarse de un estudio de asociación y no de una estimación de frecuencias la selección no probabilística tiene una menor influencia negativa, además, la inclusión de la medicación podría afectar en el modelamiento, puesto que tendría efecto directo sobre la variable de respuesta al modificar el estado de la misma y al no cumplir criterios de confusión se tendría un riesgo de sobreajuste ${ }^{(19)}$.

A pesar de las limitaciones, estos resultados son relevantes al tratarse de la población económicamente activa, la cual potencialmente estaría sometida al efecto del TDM sobre el rendimiento laboral y académico. En este contexto, este estudio no solo es una primera aproximación del tema en Perú, sino que permitirá el esbozo de políticas para la prevención primaria y secundaria del TDM en trabajadores que estudian pregrado y servirá de base para la formulación de hipótesis de nuevos estudios prospectivos que podrían establecer una relación causal. Se concluye que la baja AF se encuentra asociada a incremento de la RDM en la población estudiada, lo cual es independiente de factores sociodemográficas y académicas.

Agradecimientos: a la Dirección de la División de Estudios para Ejecutivos (EPE) de la Universidad Peruana de Ciencias Aplicadas (UPC), por facilitar el acceso a su población de estudiantes. Al Vicerrectorado de Investigación de la UPC por el apoyo para la redacción del presente artículo. Al médico Vicente Benites-Zapata (Instituto Nacional de Salud, Lima, Perú) por su revisión y sugerencias.

Conflicto de interés: los autores declaran no tener conflictos de interés.

Fuentes de financiamiento: autofinanciado

\section{REFERENCIAS BIBLIOGRÁFICAS}

1. Ferrari AJ, Somerville AJ, Baxter AJ, Norman $\mathrm{R}$, Patten SB, Vos T, et al. Global variation in the prevalence and incidence of major depressive disorder: a systematic review of the epidemiological literature. Psychol Med. 2013 Mar;43(3):471-81. doi: 10.1017/ S0033291712001511.

2. Ruiz-Grosso P, Osada J, Hoppe W, Pedraz B, Vega-Dienstmaier J. Subregistro de trastornos depresivos. Rev Peru Med Exp Salud Publica. 2011 Oct-Dec;28(4):697.

3. Burgard SA, Elliott MR, Zivin K, House JS. Working conditions and depressive symptoms: a prospective study of US adults. J Occup Environ Med. 2013 Sep;55(9):100714. doi: 10.1097/JOM.0b013e3182a299af.

4. Roca M, Gili M, Garcia-Campayo J, GarcíaToro M. Economic crisis and mental health in Spain. Lancet. 2013 Dec 14;382(9909):19778. doi: 10.1016/S0140-6736(13)62650-1

5. Reavley N, Jorm AF. Prevention and early intervention to improve mental health in higher education students: a review. Early Interv Psychiatry. 2010 May;4(2):132-42. doi: 10.1111/j.1751-7893.2010.00167.x.

6. Hong X, Li J, Xu F, Tse LA, Liang Y, Wang Z, et al. Physical activity inversely associated with the presence of depression among urban adolescents in regional China. BMC Public Health. 2009 May 20;9:148. doi: 10.1186/1471-2458-9-148.

7. Hoare E, Skouteris H, Fuller-Tyszkiewicz M, Millar L, Allender S. Associations between obesogenic risk factors and depression among adolescents: a systematic review. Obes Rev. 2014 Jan;15(1):40-51. doi: 10.1111/obr.12069.

8. van Uffelen JG, van Gellecum YR, Burton NW, Peeters G, Heesch KC, Brown WJ. Sitting-time, physical activity, and depressive symptoms in mid-aged women. Am J Prev Med. 2013 Sep;45(3):276-81. doi: 10.1016/j. amepre.2013.04.009.

9. Barcelos-Ferreira R, Nakano EY, Steffens DC, Bottino CM. Quality of life and physical activity associated to lower prevalence of depression in community-dwelling elderly subjects from Sao Paulo. J Affect Disord. 2013 Sep 5;150(2):616 22. doi: 10.1016/j.jad.2013.02.024.

10. Ströhle A. Physical activity, exercise, depression and anxiety disorders. J Neural Transm. 2009 Jun;116(6):777-84. doi: 10.1007/s00702-008-0092-x.

11. Serón P, Muñoz S, Lanas F. Nivel de actividad física medida a través del cuestionario internacional de actividad física en población Chilena. Rev Med Chile. 2010;138(10):1232-9.

12. Nebreda C, Aliaga L. Test de Depresión Mayor (Major Depression Inventory-MDI). Hillerød: Psychiatric Research Unit, Mental Health Centre North Zealand; 2003.

13. Ikenouchi-Sugita A, Yoshimura R, Sugita $\mathrm{K}$, Hori H, Yamada K, Sakaue M, et al. The effects of a walking intervention on depressive feelings and social adaptation in healthy workers. J UOEH. 2013 Mar 1;35(1):1-8.

14. Rimer J, Dwan K, Lawlor DA, Greig CA, McMurdo M, Morley W, et al. Exercise for depression. Cochrane Database Syst
Rev. 2012 Jul 11;7:CD004366. doi: 10.1002/14651858.CD004366.pub5.

15. Carek PJ, Laibstain SE, Carek SM. Exercise for the treatment of depression and anxiety. Int J Psychiatry Med. 2011;41(1):15-28.

16. Craft LL, Perna FM. The Benefits of Exercise for the Clinically Depressed. Prim Care Companion J Clin Psychiatry. 2004;6(3):104-111.

17. Heyman E, Gamelin FX, Goekint M, Piscitelli F, Roelands B, Leclair E, et al. Intense exercise increases circulating endocannabinoid and BDNF levels in humans--possible implications for reward and depression. Psychoneuroendocrinology. 2012 Jun;37(6):844-51. doi: 10.1016/j. psyneuen.2011.09.017.

18. Castillo I, Molina-García J. Adiposidad corporal y bienestar psicológico: efectos de la actividad física en universitarios de Valencia, España. Rev Panam Salud Publica. 2009 Oct;26(4):334-40.

19. Rothman, KJ, Greenland, S, Lash, TL. Modern Epidemiology. 3ra ed. Philadelphia: Lippincott Williams \& Wilkins; 2008 .

20. Bonde JP. Psychosocial factors at work and risk of depression: a systematic review of the epidemiological evidence. Occup Environ Med. 2008 Jul;65(7):438-45. doi 10.1136/oem.2007.038430.

Correspondencia: Edward Mezones-Holguin.

Dirección: Prolongación Primavera 2390,

Monterrico, Lima, Perú.

Teléfono: +51971107298

Correo electrónico: emezones@gmail.com 